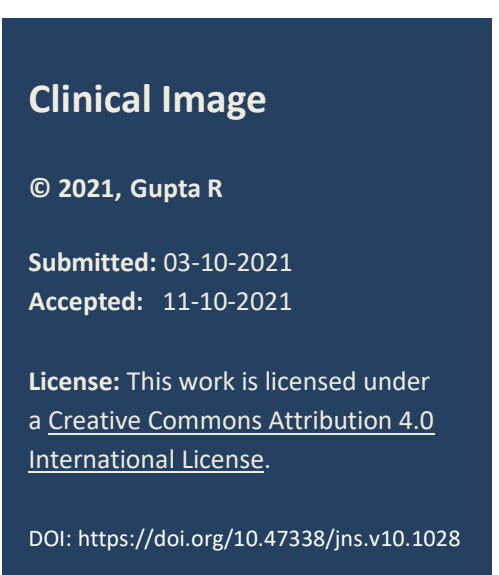

\title{
Blueberry Muffin Syndrome
}

\section{Rahul Gupta*}

Department of Paediatric Surgery, SMS Medical College, Jaipur, Rajasthan, India.

Correspondence*: Dr. Rahul Gupta, MS, M.Ch. (Paediatric Surgery) FMAS, Associate Professor, Department of Paediatric Surgery, SMS Medical College, Jaipur, Rajasthan, India. E-mail: meetsurgeon007@gmail.com

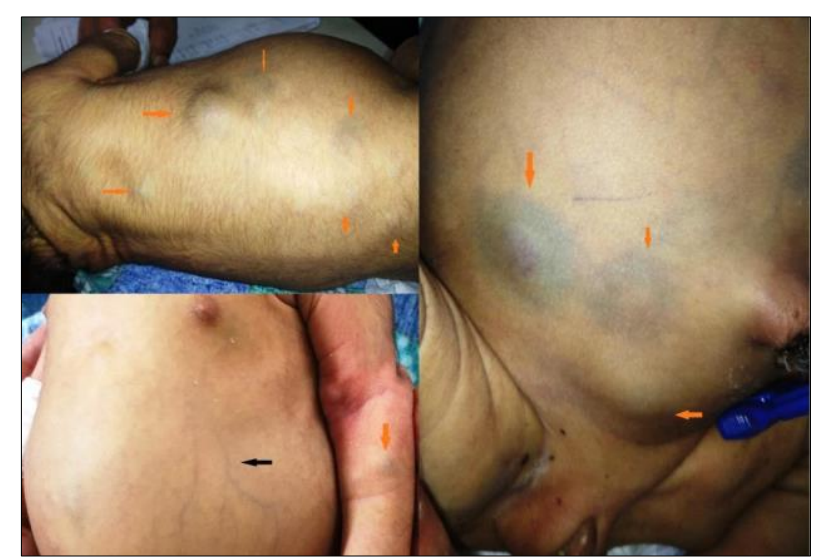

Figure 1: Photographs showing multiple blueberry muffin nodules and papules over the back (orange arrows), forearm (orange arrow), and lower abdomen (orange arrows); dilated veins visible over the abdomen (black arrow).

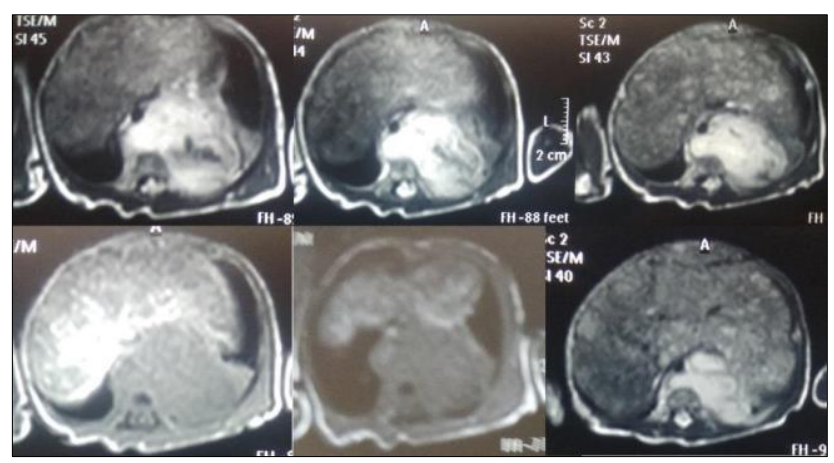

Figure 2: Photographs showing MRI abdomen (T2 transverse images) revealing left retroperitoneal, prevertebral, irregular, lobulated, hyper-intense mass, crossing the midline; mass is lifting and pushing the aorta and IVC anteriorly and extending into the posterior mediastinum. Diffuse infiltration of the liver is present ( $\mathrm{T} 1$ transverse images).

A 7-day-old full-term female neonate (weighing 2500 gm) presented with multiple purple-blue patches over the abdomen, back and face, since the second day of life (Fig. 1).The baby had gradual progressive abdominal distension and yellowish discoloration of skin since the third day of life. Antenatal history of medical treatment for Molluscum contagiosum involving the lower extremities during mid-pregnancy. The mother had a fibroid uterus with a history of intake of oral contraceptive pills. Abdominal examination revealed a distended abdomen with hepatosplenomegaly. Serum LDH levels were raised (1178 U/L) and Serum vanillylmandelic acid (VMA) was elevated. MRI abdomen revealed a left-sided retroperitoneal irregular, lobulated, hyper-intense mass, and crossing midline (Fig. 2); mass was lifting and pushing the aorta and IVC anteriorly and extending into the posterior mediastinum. It also showed gross hepatomegaly with multiple hyperintense nodular lesions, diffusely present in both the lobes of the liver; characteristic pepper pot sign present suggestive of multiple secondary lesions / infiltrative disease. A provisional diagnosis of stage 4S neuroblastoma with posterior mediastinal extension with metastatic lesions involving skin and liver was made on a clinico-radiological basis.

The term blueberry muffin baby (also known as blueberry muffin syndrome or rash or sign) was originally coined by pediatricians to describe skin appearance observed in newborns infected with rubella during the American epidemic of the 1960s.[1,2] It is characterized by generalized oval or circular-shaped, maculopapular lesions of reddish-blue or purplish-blue color.[1] These eruptions are usually seen on the trunk, head, and neck. The differential diagnoses of blueberry muffin babies consist of conditions associated with (a) prolonged dermal (extramedullary) hematopoiesis, (b) infiltrative neoplastic lesions of the skin, and (c) cutaneous vascular anomalies.[1]

Medical conditions that cause dermal hematopoiesis include intrauterine infections (TORCH complex, cytomegalovirus, rubella, neonatal lupus erythematosus) and hematologic dyscrasias (congenital spherocytosis, ABO, and Rh blood group incompatibility and anemia caused by twin-to-twin transfusion).[2,3,4] Neoplastic causes are neonatal NB, leukemia cutis 
(acute myeloid leukemia), congenital alveolar rhabdomyosarcoma, Langerhans cell histiocytosis.[1-5] Cutaneous vascular anomalies are multiple hemangiomas of infancy, multifocal lymphangioendotheliomatosis, blue rubber bleb nevus syndrome, and multiple glomangiomas.[1-5] In the maximum number of infants, the lesions evolve into light brown macules and disappear completely within a few (3 to 6) weeks. When these lesions progress and enlarge or continue to exist and do not diminish, the neoplasm is suspected; in the index case, the diagnosis was $4 \mathrm{~S}$ neuroblastoma which has a good prognosis in the majority of the patients. $[2,5]$

\section{REFERENCES}

1. Basu S, Gupta AK, Kumar A, Singh P, Kumar A. Congenital rubella syndrome presenting at birth with blueberry muffin rash and communicating hydrocephalus. J Pediatr Neurol. 2009; 7:423-6.

2. Mehta V, Balachandran C, Lonikar V. Blueberry muffin baby: A pictorial differential diagnosis. Dermatol Online J. 2008; 14:8

3. Hsiao YW, Tseng FW, Shih YL, Kuo TT, Jiang TH, Hui CY. Blueberry muffin baby with acute myeloid
Acknowledgements: I am sincerely thankful to the Faculty and residents of the department of Paediatric Surgery, Department of Radiodiagnosis (especially Dr. Anu Bhandari for their guidance), and Dr. Manisha Goyal (Medical Geneticist), Department of Paediatrics, SMS Medical College, Jaipur, Rajasthan, India.

Conflict of Interest: None declared

Source of Support: Nil

Consent to Publication: Author(s) declared taking informed written consent for the publication of clinical photographs/material (if any used), from the legal guardian of the patient with an understanding that every effort will be made to conceal the identity of the patient, however it cannot be guaranteed.

Author Contributions: Author(s) declared to fulfil authorship criteria as devised by ICMJE and approved the final version.

leukemia and spontaneous remission. Dermatologica Sin. 2011; 29:47-9.

4. van Ijsselmuiden MN, Bekhof J, Peek AM. A neonate with the 'blueberry muffin syndrome'. Ned Tijdschr Geneeskd. 2014; 157:A6460.

5. Isaacs H Jr. Cutaneous metastases in neonates: a review. Pediatr Dermatol. 2011; 28:85-93. 